\title{
Paradigm Changes and the Future of HIV Vaccine Research: A Summary of a Workshop Held in Baltimore on 20 November 2013
}

Marc HV Van Regenmortel ${ }^{1 *}$, Jean-Marie Andrieu², Dimiter S Dimitrov ${ }^{3}$, Barbara Ensoli ${ }^{4}$, Catarina E Hioe ${ }^{5}$, Christiane Moog ${ }^{6}$ and Ruth M Ruprecht ${ }^{7}$

${ }^{1}$ CNRS, School of Biotechnology, University of Strasbourg, France

2Université Paris Descartes, Paris, France

${ }^{3}$ National Cancer Institute, NIH, Frederick, Maryland, USA

${ }^{4}$ National AIDS Center, IstitutoSuperiore di Sanita, Rome, Italy

${ }^{5}$ New York University School of Medicine, New York, USA

6INSERM Unit 1110, University of Strasbourg, France

${ }^{7}$ Texas Biomedical Research Institute, San Antonio, Texas, USA

\begin{abstract}
A workshop entitled: "What type of HIV Vaccine research should be promoted" took place during a virology conference held in Baltimore in November 2013 (www.omicsgroup.com/conferences/virology-2013/). The purpose of this workshop was to discuss new paradigms that better fit our increased knowledge of HIV immunopathology and which could possibly be more helpful in guiding future vaccine research than did past unsuccessful approaches. Panelists were asked to respond to four questions regarding possible novel paradigms that could guide future HIV vaccine research and their responses are summarized.
\end{abstract}

Keywords: HIV vaccines; Paradigms; Reverse vaccinology; Antibody maturation; Immunogenicity; Tat vaccine; Research funding

\section{Introduction}

The concept of scientific paradigm was made popular by Thomas Kuhn in his influential book "The Structure of Scientific Revolutions" published in 1962 [1]. He argued that scientists in their research are always guided by assumptions and theoretical presuppositions that determine the lines of investigation they pursue. These underlying assumptions which often are not explicitly stated represent the prevailing paradigms present in every field of scientific enquiry at any particular time. The field of HIV vaccine research is no exception and several paradigms have had a major influence on the type of research undertaken and funded in the last 25 years [2]. Since investigators are not always fully aware of the underlying paradigms that influence their choice of research program and experimental approach, they may not question the validity of a particular paradigm when they regularly obtain results that are not consistent with the hypothesis or theory that gave rise to the paradigm. When this happens, they may continue to pursue unfruitful lines of investigation that impede scientific progress. This pitfall can only be avoided if researchers remain constantly aware of the paradigms that make them pursue a particular research program.

In HIV vaccine research, there is evidence that several prevalent paradigms have not helped the development of the field, and this may partly explain why after 25 years of intensive research efforts, it has not been possible to develop an effective vaccine using classical strategies [3-6]. One such paradigm is the assumption that HIV-1 epitopes identified by crystallography of complexes of HIV Env bound to affinity -matured neutralizing (n) Mabs are likely to be effective vaccine immunogens able to induce a protective immune response. Another misleading paradigm is the assumption that anti-HIV-1 Mabs are monospecific for a single viral epitope instead of always being polyspecific and able to bind numerous epitopes different from the one identified when the structure of the Mab-HIV-1 complex was solved. A third one is the expectation that after isolating from HIV-1 infected individuals increasing numbers of nAbs that may be useful for passive immunotherapy, this will necessarily facilitate the development of immunogens suitable for active immunization [7].

\section{A New Paradigm Advocating Basic Research}

Paradigms come in different forms and some correspond to general conceptual frameworks and theoretical assumptions that permeate an entire field at a particular time. In recent years many HIV investigators have become convinced that our current knowledge of the human immune system and of HIV-1 immunopathology is far too limited to permit the development of an HIV-1 vaccine in the near future. This has given rise to a new popular paradigm which assumes that this stumbling block to vaccine development can only be overcome by embarking on large scale basic research programs in immunology $[8,9]$. This paradigm espouses the classic thesis of Vannevar Bush, prevalent since the end of the Second World War [10], which claims that all technological innovations are derived from applied research programs that always find their origin in curiosity-driven basic research performed without any consideration of potential practical use. This thesis is no longer universally accepted since the separation between basic and applied research is nowadays often perceived as somewhat of a false dichotomy. A more realistic account of scientific and technological progress proposed by Stokes [11] follows the so-called quadrant model of scientific research which accepts that research can be driven simultaneously by a quest for fundamental understanding and by considerations of use for solving a practical problem. According to this model, a commitment to try to understand HIV-1 immunopathology need not exclude a commitment to try to control HIV-1 infection

*Corresponding author: Marc HV Van Regenmortel, CNRS, UMR7242-Institut de Recherche de l'Ecole de Biotechnologie de Strasbourg (IREBS), Université de Strasbourg, Illkirch 67400, France, Tel: 330388 312485; E-mail: vanregen@unistra.fr

Received January 15, 2014; Accepted February 18, 2014; Published February 25, 2014

Citation: Van Regenmortel MHV, Andrieu JM, Dimitrov DS, Ensoli B, Hioe CE, Moog C, et al. (2014) Paradigm Changes and the Future of HIV Vaccine Research: A Summary of a Workshop Held in Baltimore on 20 November 2013. J AIDS Clin Res 5: 281. doi:10.4172/2155-6113.1000281

Copyright: (c) 2014 Van Regenmortel MHV, et al. This is an open-access article distributed under the terms of the Creative Commons Attribution License, which permits unrestricted use, distribution, and reproduction in any medium, provided the original author and source are credited. 
by vaccination [12]. The remaining difficulty, of course, lies in the impossibility to predict which items of new knowledge derived from basic or applied research will subsequently allow the development of a useful technological product such as a vaccine.

\section{From Basic and Applied Research to Technological Innovation}

Two types of human knowledge are usually distinguished [13] corresponding to:

1) Knowing what is the case, for instance the existence of a natural biological phenomenon. This is usually called propositional knowledge which comprises inter alia all the scientific facts which can be expressed as true statements or propositions that such and such is the case.

2) Knowing how to do something while achieving something of practical utility, using what is called procedural or prescriptive knowledge.

Any addition to propositional knowledge is a discovery that unearths something that existed all along but was unknown to anybody. Any addition to prescriptive knowledge, on the other hand, is an invention derived from basic and applied research that makes it possible to do something that was previously unfeasible and that achieves a desirable, practical or societal goal.

An example of such a technological innovation would be an effective preventive HIV-1 vaccine that allows nature to be manipulated successfully by providing protection against viral infection. Unfortunately it is impossible to predict which item of propositional knowledge is required to lead to the prescriptive knowledge needed to manipulate and control a natural phenomenon such as a viral infection. As pointed out by Ian Hacking in his book Representing and Intervening [14], it is only by intervening in a material system that we learn to manipulate and control it, thereby deriving the desired prescriptive knowledge. For instance, it is only by comparing various ways of manipulating the human immune system, using trial-and-error empirical experimentation, that we may eventually learn to control it and achieve protective immunity by vaccination [15]. Whereas elements of propositional knowledge (for instance the 3D structure of an antibody) may be "right" or "wrong",this is not the case with the ability to manipulate the immune system using prescriptive knowledge which is either "successful" or "unsuccessful", the outcome being adjudicated empirically. This means that we achieve useful prescriptive knowledge of the immune system only by a prior successful empirical intervention that would lead for instance to protective immunity. This conclusion is at odds with the currently fashionable paradigm that increasing our knowledge of basic immunology on its own would be sufficient to improve our ability to develop an effective HIV-1 vaccine [4].

A workshop entitled: "What type of HIV vaccine research should be promoted" took place during a virology conference held in Baltimore in November 2013 (www.omicsgroup.com/conferences/virology-2013/). The purpose of this workshop was to discuss new paradigms that better fit our increased knowledge of HIV-1 immune responses and which could therefore be more helpful in guiding future vaccine research than did past unsuccessful paradigms.

In order to structure the discussion, panelists were asked to respond to four questions. Some of their responses are summarized below.

\section{Question 1: Which new ideas, hypotheses and paradigms should be introduced in the HIV-1 vaccine field?}

1) Since neutralizing antibodies and cytotoxic $T$ cells do not prevent HIV infections nor control viral replication in humans, many virologists believe that classical vaccination approaches will not succeed in the case of HIV-1. In other words, they accept that they need to achieve something that the human immune system is normally not capable of doing when it encounters the virus. Earlier attempts using the approach known as reverse vaccinology $[16,17]$ failed when the putative $\mathrm{nAb}$ germline predecessors were found to bind poorly or not at all to the HIV-1 epitopes that are recognized by their hypermutated mature descendants isolated from HIV-1 infected individuals [15]. The resulting very poor immunogenicity of HIV-1 epitopes usually prevents the human immune system to initiate and sustain a response that leads to the elicitation of broadly neutralizing (bn)Abs. Even if an immune response is initiated, a complex and lengthy antibody maturation pathway that differs in individual vaccinees is needed for obtaining bnAbs [18]. New strategies are therefore needed to identify candidate vaccine immunogens that bind germline predecessors of bnAbs or intermediates in the maturation process of these Abs. It is not clear at present whether the unravelling of individual antibody maturation pathways will succeed in identifying HIV-1 immunogens capable of inducing suitable predecessors of known bnAbs in populations of naïve individuals.

2) New strategies should be introduced to identify the immunogens responsible for $\mathrm{Ab}$ responses associated with vaccine protection. One such approach called protection-linked biopanning, using recombinant phages encoding random peptide libraries, was found to be able to identify viral epitopes that bind to antibodies present only in vaccinated protected individuals [19]. Earlier paradigms unfortunately led investigators to concentrate on epitopes that only bind bnMabs (i.e. on viral antigens) rather than on immunogens containing immunogenic epitopes capable of eliciting such antibodies [15].

3) Since most HIV-1 infections occur through sexual contact, more studies should be devoted to mechanisms of mucosal immunity. Little is known about how HIV-1 disseminates through the mucosa. If dendritic cells are involved, Fc-mediated inhibition could interfere with the infection process. If the first target is a CD4 T lymphocyte, preventing infection of CD4 T cells should be a priority. Since cells at mucosal surfaces express various Fc receptors, immune complex trapping through these receptors may lead either to virus degradation by phagocytosis or to enhancement of virus replication in the case of neonatal receptors. Studies aimed at developing immune responses at mucosal surfaces using specific adjuvants or involving certain bacterial flora also deserve further investigation.

4) Although IgA represents the most abundant immunoglobulin made by the human body and is an important component of mucosal secretions, the role of the various IgA forms in either preventing or enhancing HIV transmission is unclear. Only one study thus far has examined the difference between IgA1 and IgA2 present mucosally as dimers [20]. In this study, dimeric IgA1 was significantly more protective than the dimeric IgA2 version of a neutralizing anti-HIV $\mathrm{mAb}$ with the same epitope specificity. In contrast, serum IgA responses against the HIV envelope have been linked with an increased risk of virus acquisition in the RV144 trial. The fact that IgA, in different forms and from different anatomical compartments, has been associated with either protection or increased risk of virus acquisition, indicates that the role of serum and/or mucosal IgA in preventing or facilitating HIV transmission should be further investigated. The role of mucus and 
natural mucosal fluids, in addition to mucosal antibodies, in enhancing or decreasing virion capture should also be examined.

5) Although replicating vectors have scored the best at inducing long-term memory, these vectors are mostly attenuated pathogens and since their long-term safety may be a serious issue, alternative strategies using for instance intradermal injections and intranasal administration [21] should be studied.

6) Currently available animal models are not satisfactory to allow large numbers of candidate vaccines to be evaluated. In order to test more vaccine candidates and strategies, additional cost-effective highthroughput animal models that are able to be transposed to generate appropriate human immune responses should be developed. The development of novel virus chimeras using Env from different virus clades, similar to SHIV in macaques, should also be encouraged.

7) Since SIV in macaques and HIV-1 in humans can only replicate efficiently in vivo if mucosal CD4 T cells are activated, it has been hypothesized that it might be possible to suppress viral replication by interfering with CD4 T cell activation. This was tested by stimulating mucosal dendritic cells in macaques with a mucosal vaccine containing inactivated SIV associated with the Calmette-Guerin bacillus, in an attempt to induce in vivo the terminal differentiation of SIV-specific CD8 T cells. This mucosal vaccination was found to be extremely effective since after intrarectal challenges with large amounts of SIV, most macaques remained sterilely protected. The protection was found to be induced by CD8 $\mathrm{T}$ cells that possessed strong SIV suppressive activity, and surprisingly it was not associated with SIV-specific antibodies or CTLs (unpublished results by Andrieu JM and Lu $\mathrm{W})$. This led to the further hypothesis that this type of vaccine may have acted through a phenomenon of mucosal/oral tolerance. Since probiotic bacteria, for instance lactobacilli, have been suspected for a long time to be inducers of immune tolerance, an oral vaccine made with the same killed SIV immunogen in association with large amounts of Lactobacillus plantarum was tested in macaques. This vaccine was found to induce a previously unrecognized class of non-cytolytic MHC 1b/E restricted CD8 regulatory T cells (Tregs) which specifically suppressed the activation of SIV positive CD4 T lymphocytes. This suppression which prevented the initial burst of virus replication in vivo, permanently protected 15 out of 16 macaques from infection [22]. This strong protective effect induced by CD8 + Tregs was obtained in experiments that were based on an "out of the box" innovative paradigm, confirming the assumption that SIV requires activated immune cells for its replication. Since CD $4+T$ cell activation drives both the initial SIV and HIV-1 replication in macaques and humans respectively, this approach is currently being tested in humans. If successful it could offer an exciting prospect for a preventive or therapeutic HIV-1 vaccine.

8) In recent years, considerable and growing attention has been given to the development of therapeutic HIV vaccines for treating people already infected with HIV-1. Immunization with the conserved HIV regulatory protein, Tat, has been shown to stimulate the immune system of patients receiving antiretroviral therapy, leading to a further and stable CD4 T cell increase and immune restoration [23]. Extracellular HIV-1 Tat can form a molecular complex with trimeric Env, shielding it from anti-Env nAbs and redirecting virus entry to RGD-binding integrins [24]. Anti-Tat Abs, which are infrequently produced upon natural infection, are able to restore and increase HIV neutralization that would normally be impaired by extracellular Tat [24]. Since vaccination with Tat decreases the proviral DNA load (Ensoli et al. submitted), slows down the progression to AIDS and can lead to complete or partial protection from infection [19,24-26], the accumulated evidence supports a novel paradigm that views HIV-1 Tat as an important vaccine candidate either on its own or as part of a multicomponent vaccine [19,24-26].

\section{Question 2: How should innovative research on HIV vaccines be funded, especially risky projects that are unlikely to be favored by selection committees because of insufficient confirmed data?}

9) The current prevalent funding structure focusing predominantly on large networks of investigators under strong leadership of a principal investigator has considerable built-in inertia which makes it difficult to rapidly adapt to new paradigms. Generous funding of smaller groups and networks would allow the pursuit of more flexible lines of research based on original paradigms and would foster greater intellectual flexibility. Currently, at most $10 \%$ of available funding is devoted to supporting high-risk projects based on innovative paradigms. Funding redistribution should be encouraged and a significantly larger funding percentage (perhaps up to 50\%) should be used for smaller scale innovative projects. Such a change would diversify the vaccine approaches that can be investigated and would increase the probability of finding an effective vaccine.

10) It can be argued that the reigning paradigms followed in the past by well-funded large networks of investigators were usually nothing more than hypothetical assumptions for which very limited confirmed HIV data existed. Since it is impossible to predict which proposed experimental approaches will later be found to be empirically successful, funding agencies should utilize selection committees comprising accomplished open-minded experts who themselves have made original contributions outside the framework of conservative, conventional wisdom. The validity of human judgments cannot easily be quantified and it is questionable whether the majority vote of a large selection committee will necessarily back the most promising and original proposal rather than projects that follow the accepted scientific consensus.

11) High-risk projects based on novel paradigms should be examined by selection committees different from those in charge of traditional four year grants requiring extensive supporting data and a solid track record of publications. Many traditional grants support fundamental immunological research that is considered to be of high quality because it produces excellent scientific papers published in high impact journals such as Science, Nature, Cell, Journal of Virology etc. These papers tend to include statements claiming that the newly acquired scientific information is likely to help the future development of an HIV-1 vaccine, although they rarely present new prescriptive knowledge relevant to the goal of producing a technological innovation in the form of an effective vaccine. Projects considered to be risky because they follow innovative, unorthodox paradigms that lack widespread support could be funded by two-phase grants, first to establish that they do produce prescriptive knowledge of potential utility, and then subsequently transferring the pilot study to a larger study. However, the emphasis should be on useful prescriptive knowledge relevant to vaccines rather than to curiosity-driven basic research that only increases our immunological knowledge.

12) Innovative projects could also be funded by a procedure similar to that used by the MacArthur Foundation (http://www.macfound. org). Funding would be allocated for a period of several years and a comprehensive report would only have to be produced at the end of the contract and not annually. Applicants should demonstrate high levels of past creativity and accomplishments in vaccinology and related fields 
and the allocated funding should suffice for carrying out small phase I or phase II clinical trials.

13) The surprising extensive protection against SIV achieved in macaques with an oral tolerogenic vaccine that did not elicit SIVspecific antibodies nor CTLs was obtained by an investigator-driven research project that was not funded by a governmental or large scale international research organization [22]. It was entirely sponsored by a private benefactor who agreed to fund a risky project based on an unorthodox paradigm for which minimal preliminary data existed. This illustrates the benefits that sometimes accrue from moving away from reigning, fashionable paradigms.

Question 3: What should be the balance between empirical vaccine research (for instance testing various hypotheses on how to induce protective immunity) and so-called "rational" vaccine design based on structural knowledge?

14) Empirical and rational approaches to vaccine development are sometimes opposed as if they were incompatible and it has for instance been claimed that "Rational design represents the only approach that can elevate vaccine research from an empirical exercise to a scientific discipline" [27]. However, all existing vaccines have been developed using trial-and-error immunization trials that are entirely rational and there is no example of an effective vaccine ever having been obtained solely by rational design based on structural analysis [7]. Claims to the contrary arise because the structure-based rational design of a viral epitope engineered to better fit a single bnMab is masquerading as vaccine immunogen design, a confusion which is due to the erroneous assumption that antigen binding reactivity necessarily entails an immunogenic capacity to induce protective Abs [15]. As discussed above, structural knowledge of viral epitopes and antibody paratopes corresponds to propositional knowledge which does not tell us how to successfully manipulate the immune system so that immunization with an appropriate immunogen will elicit a protective anti-viral response. Only after an effective immunogen has been identified empirically does it become possible to investigate its possible mode of action. This should involve a study not only of possible neutralizing antibodies but should include the study of barrier functions at mucosal surfaces, virion trapping properties of mucosal secretions and cellular as well as innate immunity phenomena that may prevent cell-to-cell virus transmission in primary and chronic infections. Developing an effective vaccine empirically is therefore the first priority and should precede attempts at elucidating the theoretical mode of action of an ideal HIV-1 vaccine on the basis of our knowledge of the immune system.

15) The lack of success of the reverse vaccinology/engineering paradigm should be acknowledged as such and should lead to a paradigm shift emphasizing the relevance of the bnAb germline ancestors and their maturation. In 2013, Bruce Alberts, the Editor of Science wrote the following: "Scientists need to develop a value system where simply moving on from one's mistakes without publicly acknowledging them, severely damages, rather than protects, a scientific reputation." Knowing what leads researchers astray is as important to science as knowing which hypothesis is corroborated by experimental observations.

\section{Question 4: If you had the authority to do it, what type of "out} of the box" vaccine concepts would you support for funding?

16) In view of the high level of somatic hypermutation observed in all anti-HIV-1 bnAbs, any studies addressing the maturation pathways of such Abs should be encouraged. There is, however, no guarantee that studying stochastic maturation processes in individual immune systems would provide the prescriptive knowledge required for reproducing similar maturation phenomena in large populations of vaccinees.

17) Methods for inducing strong mucosal antibody responses involving dimeric or multimeric IgA1 should be investigated. New immunogens and adjuvants should be tested and the role of various $\mathrm{Ab}$ inhibitory functions in protection should be analyzed.

18) More studies of HIV-1 immunopathogenesis should be undertaken in an attempt to identify potential key targets for intervention as well as new biomarkers of disease progression and of infection control. Other markers than viral load and CD4 T cells counts are needed to assess vaccine efficacy, for instance proviral DNA load, cell-to-cell transmission, different neutralization protocols, functional $\mathrm{T}$ and $\mathrm{B}$ cell subsets etc.

19) In view of the totally unexpected "out of the box" results observed in macaques orally vaccinated with killed SIV plus Lactobacillus plantarum, a duplication trial in the macaque model and subsequently a phase one clinical study in humans should be performed as soon as possible. If the hypothesis of Andrieu and $\mathrm{Lu}$ [22] is confirmed in such simian and human experiments, considerable funding should be engaged to advance its possible clinical development.

\section{Acknowledgements}

M. Van Regenmortel is grateful to Alexandra Brown for typing the manuscript and to Florence Diemer for efficient secretarial assistance.

\section{References}

1. Kuhn TS (1962) The Structure of Scientific Revolutions. University of Chicago Press.

2. Esparza J (2013) What has 30 years of HIV research taught us? Vaccines 1 513-526.

3. Van Regenmortel MHV (2011) Limitations to the structure-based design of HIV1 vaccine immunogens. J MolRecogn 24: 741-753.

4. Van Regenmortel MHV (2012) An Introduction to the current state of HIV vaccine research. J AIDS Clinic Res S8: e001.

5. Burton DR, Ahmed R, Barouch DH, Butera ST, Crotty S, et al. (2012) A blueprint for HIV vaccine discovery. Cell Host Microbe 12: 396-407.

6. Esparza J (2013) A brief history of the global effort to develop a preventive HIV vaccine. Vaccine 31: 3502-3518.

7. Van Regenmortel MHV (2012) Requirements for empirical immunogenicity trials, rather than structure-based design, for developing an effective HIV vaccine. Arch Virol 157: 1-20.

8. Mc Elrath MJ, Haynes BF (2010) Induction of immunity to human immunodeficiency virus type 1 by vaccination. Immunity 33: 542-554.

9. Virgin HW, Walker BD (2010) Immunology and the elusive AIDS vaccine. Nature 464: 224-231.

10. Bush V (1945) Science - The Endless Frontier: A Report to the President on a Program for Hostwar Scientific Research. Washington: National Science Foundation.

11. Stokes DE (1997) Pasteur's Quadrant. Basic Science and Technologica Innovation. Brooking Institution Press, Washington, DC.

12. Esparza J (2005) The global HIV vaccine enterprise. International Microbiology 8: $93-101$

13. Mokyr J (2002) Technology and the Problem of Human Knowledge. In: The Gifts of Athena. Historical origins of the Knowledge Economy. Princeton University Press, Princeton, 1-27.

14. Hacking I (1983) Representing and Intervening. Cambridge University Press Princeton.

15. Van Regenmortel MHV (2012) Basic research in HIV vaccinology is hampered by reductionist thinking. Front Immunol 3: 194. 
Citation: Van Regenmortel MHV, Andrieu JM, Dimitrov DS, Ensoli B, Hioe CE, Moog C, et al. (2014) Paradigm Changes and the Future of HIV Vaccine Research: A Summary of a Workshop Held in Baltimore on 20 November 2013. J AIDS Clin Res 5: 281. doi:10.4172/2155-6113.1000281

Page 5 of 5

16. Burton DR (2002) Antibodies, viruses and vaccines. Nat Rev Immunol 2: 706713.

17. Van Regenmortel MHV (2011) Two meanings of reverse vaccinology and the empirical nature of vaccine science. Vaccine 29: 7875.

18. Dimitrov DS (2010) Therapeutic antibodies, vaccines and antibodyomes. Mabs 2: $347-356$.

19. Bachler BC, Humbert M, Palikuqi B, Siddappa NB, Lakhashe SK, et al. (2013) Novel biopanning strategy to identify epitopes associated with vaccine protection. J Virol 87: 4403-4416.

20. Watkins JD, Sholukh AM, Mukhtar MM, Siddappa NB, Lakhashe SK, et al. (2013) Anti-HIV IgA isotypes: differential virion capture and inhibition of transcytosis are linked to prevention of mucosal R5 SHIV transmission. AIDS 27: F13-F20.

21. Bomsel M, Tudor D, Drillet AS, Alfsen A, Ganor Y, et al. (2011) Immunization with HIV-1 gp41 subunit virosomes induces mucosal antibodies protecting nonhuman primates against vaginal SHIV challenges. Immunity 34: 269-280.

22. Lu W, Chen S, Lai C, Guo W, Fu L, et al. (2012) Induction of CD8+ regulatory T cells protects macaques against SIV challenge. Cell Reports 2: 1736-1746.
23. Ensoli B, Bellino S, Tripiciano A, Longo O, Francavilla V, et al. (2010) Therapeutic immunization with HIV-1 Tat reduces immune activation and loss of regulatory T-cells and improves immune function in subjects on HAART. PLoS ONE 5: e13540.

24. Monini P, Cafaro A, Srivastava IK, Moretti S, Sharma VA, et al. (2012) HIV-1 Tat promotes integrin-mediated HIV transmission to dendritic cells by binding Env spikes and competes with neutralization by anti-HIV antibodies. PLOS ONE 7 : e48781.

25. Cafaro A, Caputo A, Fracasso C, Maggiorella M T, Goletti D, et al. (1999) Control of SHIV-89.6P-infection of cynomolgus monkeys by HIV-1 Tat protein vaccine. Nat Med 5: 643-650.

26. Cafaro A, Bellino S, Titti F, Maggiorella M T, Sernicola L, et al. (2010) Impact of viral dose and major histocompatibility complex class IB haplotype on viral outcome in Mauritian Cynomolgus monkeys vaccinated with Tat upon challenge with simian/human immunodeficiency virus SHIV89.6P. J Virol 84: 8953-8958.

27. Karlsson Hedestam GB, Fouchier RA, Phogat S, Burton DR, Sodroski J, et al. (2008) The challenges of eliciting neutralizing antibodies to HIV-1 and to influenza virus. Nat Rev Microbiol 6: 143-155. 